\title{
Bur open Intensity versus duration of physical activity: implications for the metabolic syndrome. A prospective cohort study
}

\author{
Adam Hoegsbro Laursen, ${ }^{1,2}$ Ole P Kristiansen, ${ }^{1}$ Jacob Louis Marott, ${ }^{2}$ \\ Peter Schnohr, ${ }^{2}$ Eva Prescott ${ }^{1,2}$
}

To cite: Laursen $\mathrm{AH}$, Kristiansen OP, Marott JL, et al. Intensity versus duration of physical activity: implications for the metabolic syndrome. A prospective cohort study. BMJ Open 2012;2: 001711. doi:10.1136/bmjopen-2012001711

- Prepublication history for this paper are available online. To view these files please visit the journal online (http://dx.doi.org/10.1136/ bmjopen-2012-001711).

Received 25 June 2012 Accepted 21 August 2012

This final article is available for use under the terms of the Creative Commons Attribution Non-Commercial 2.0 Licence; see http://bmjopen.bmj.com

${ }^{1}$ Department of Cardiology, Bispebjerg University Hospital, Copenhagen, Denmark

${ }^{2}$ The Copenhagen City Heart Study, Bispebjerg University Hospital, Copenhagen, Denmark

Correspondence to Dr Eva Prescott; epre0004@bbh.regionh.dk

\section{ABSTRACT}

Objectives: To explore the relative importance of leisure time physical activity (LTPA), walking and jogging on risk of developing the metabolic syndrome (MS).

Design: A prospective cohort study.

Setting: The Copenhagen City Heart Study.

Participants: 10135 men and women aged 21-98 years who attended an initial examination in 1991-1994 and were re-examined after 10 years.

Outcome measures: The association of LTPA, jogging, walking speed and walking volume with MS at baseline and at 10-year follow-up was investigated by multiple logistic regression analyses.

Results: Baseline prevalence of MS was $20.7 \%$ in women and $27.3 \%$ in men. In both women and men, MS prevalence was associated with lower LTPA and walking speed and was lower in joggers compared to non-joggers. In subjects free of MS at baseline, 15.4\% had developed MS at 10-year follow-up. Risk of developing MS was reduced in subjects with moderate or high LTPA, higher walking speed and in joggers whereas a higher volume of walking was not associated with reduced risk. After multiple adjustment, odds ratio (OR) of developing MS in moderate/high LTPA was 0.71 $(95 \% \mathrm{Cl} 0.50$ to 1.01$)$, fast walking speed 0.51 ( 0.33 to 0.80 ) and joggers $0.60(0.37$ to 0.95$)$ and walking $>1 \mathrm{~h}$ daily 1.22 (0.91 to 1.65$)$.

Conclusions: Our results confirm the role of physical activity in reducing MS risk and suggest that intensity more than volume of physical activity is important.

\section{INTRODUCTION}

The metabolic syndrome (MS) is a condition of accumulated risk factors of metabolic origin. ${ }^{2}{ }^{2}$ The risk factors include central obesity, hypertension, insulin resistance, impaired glucose tolerance and dyslipidaemia. ${ }^{1-6}$ Moreover, the MS is characterised by a prothrombotic and a proinflammatory state. ${ }^{17}$ There is an ongoing discussion on whether MS represents a disease in itself or merely a cluster of risk factors. ${ }^{5} 8$ Regardless, abdominal obesity ${ }^{1}$ and insulin resistance ${ }^{19}$

\section{ARTICLE SUMMARY}

Article focus

- Physical activity reduces cardiovascular risk partly through the factors clustered in the metabolic syndrome.

- It is unclear whether longer duration or higher intensity of exercise is more efficient in combating the metabolic syndrome.

Key messages

- On the basis of a cohort study with repeated examinations, this study finds that intensity of daily exercise such as fast walking and jogging reduce the risk of developing the metabolic syndrome by as much as $50 \%$ whereas walking as much as $1 \mathrm{~h}$ daily is not associated with reduced risk.

- This information contributes to improve recommendations on daily exercise.

Strengths and limitations of this study

- Study strengths include the considerable study size, the unselected study sample and the longitudinal study design with repeated measures allowing us to assess the causal inference between volume and intensity of exercise and the metabolic syndrome.

- Some limitations are the questionnaire-based information on level of leisure time physical activity and lack of direct information on fitness.

play key roles in MS and a sedentary lifestyle along with dietary and genetic factors are thought to be implicated in the pathogenesis. ${ }^{4} 6$

The high prevalence of MS in different populations ${ }^{7}$ has led to several studies exploring the association between physical activity and MS risk. ${ }^{3} 46$ 10-13 Cross-sectional analyses have suggested that physical activity may prevent obesity as well as insulin resistance potentially reducing risk of MS development. ${ }^{4}{ }^{7} 14$ Similarly, cross-sectional studies have documented an inverse dose-response relationship between physical activity in 
leisure time and MS risk. ${ }^{36}$ However, cross-sectional analyses limit causal inference making it impossible to determine whether a low physical activity level is the result of obesity as a central component of MS or if the MS develops as a consequence of physical inactivity. A handful of prospective studies have investigated the link and results have been conflicting. ${ }^{9} 10$ 15-17

Recent studies indicate that intensity rather than volume of physical activity is important to reduce cardiovascular risk. ${ }^{18-22}$ Several of these studies have used walking volume (duration) and speed (intensity) and potential mechanisms are through improved metabolic fitness. The aim of this study was therefore to examine the effect of leisure time physical activity (LTPA) on MS risk by combining a cross-sectional and a longitudinal study design and further to provide information on the relative importance of intensity and volume of physical activity.

\section{MATERIALS AND METHODS}

The study is based on data from the Copenhagen City Heart Study (CCHS), a sex-stratified and age-stratified random sample of people living within a defined area of Copenhagen initiated in 1976 with follow-ups conducted in 1981-1983, 1991-1994 and 2001-2003. The crosssectional analysis in the first part of the present study is based on data from the 10135 people (5698 women, response rate $61.2 \%$ ) aged 21-98 years participating in the CCHS3 (1991-1994). Of these, 2140 subjects died in the 10-year span between CCHS3 and CCHS4 (2001-2003) leaving 7995 subjects from CCHS3 who were invited to participate in CCHS4. However, 1692 were excluded from the longitudinal analysis due to having MS at baseline and 215 subjects were excluded due to missing values and lack of MS classification. Of the remaining 6088 participants free of MS at baseline, $3992(65.6 \%)$ participated in the fourth survey.

Physical activity habits were assessed by selfadministered questionnaires originally constructed by Saltin and Grimby ${ }^{23}$ and slightly modified for the CCHS. Questionnaires included LTPA, walking and jogging habits. We conceptualised volume of physical activity by LTPA and hours of walking, and intensity of physical activity as speed of walking and jogging. LTPA was classified as (1) Sedentary: sedentary or light physical activity (PA) (eg, slow walking/biking, light gardening work) $<2 \mathrm{~h} /$ week, (2) Light PA: light PA 2-4 h/week, (3) Moderate PA: light $\mathrm{PA}>4 \mathrm{~h} /$ week or $2-4 \mathrm{~h} /$ week of more vigorous PA (eg, brisk walking, fast biking, heavy gardening work, sports that cause perspiration or exhaustion) and (4) High PA: more than $4 \mathrm{~h} /$ week of moderate PA or regular heavy exercise or competitive sports several times per week. Thus, LTPA integrates volume and intensity with level 2 reflecting primarily volume, level 4 primarily intensity and level 3 both. Categories 3 and 4 include structured exercise. Walking volume was reported as hours/day (h/day) in three categories: $0-0.5,0.5-1.0$ and $>1 \mathrm{~h} /$ day. Walking speed was self-reported as slow, average, fast and very fast and subjects were asked to define themselves as joggers or non-joggers. To avoid inadequate group sizes the fast and very fast walking speed categories were merged. Similarly, in longitudinal analyses LTPA groups 3 and 4 were merged.

Smoking habits were classified in two categories (smokers/non-smokers), weekly alcohol consumption in four categories $(0,1-7,8-21$ and >21 units/week). Socio-economical variables included duration of schooling (0-7, 8-11 and >11 years), completed education (four categories), household income (four categories) and cohabitation (living alone or living with spouse/ partner or others).

Height and bodyweight were measured with the subjects wearing light indoor clothing and no shoes. BMI was calculated as the weight $(\mathrm{kg})$ per height squared $\left(\mathrm{m}^{2}\right)$. Waist circumference (WC) was measured to the nearest millimetre by educated staff with the subjects standing with relaxed breathing. The measuring tape was placed directly on the bare skin midway between the lower rib margin and the iliac crest. Blood pressure was measured on the left arm in a sedentary position after at least 5 min rest (a London School of Hygiene sphygmanometer was used at all examinations). Non-fasting blood samples were analysed for plasma levels of highly sensitive $\mathrm{C}$ reactive protein, fibrinogen, high-density lipoprotein (HDL) cholesterol, triglycerides and glucose.

The MS status was defined according to the latest criteria from the American Heart Association. ${ }^{2}$ However, we employed a modified version with a different cut point for plasma glucose since only non-fasting values were available. The cut-off point for triglycerides was not changed since recent research has shown only minor differences between fasting and non-fasting triglyceride values. $^{24} \mathrm{MS}$ diagnostic criteria included central obesity (WC $>102 \mathrm{~cm}$ in men, $>88 \mathrm{~cm}$ in women), elevated triglycerides $(\geq 1.7 \mathrm{mM}$ or specific drug treatment for this lipid abnormality), reduced HDL-cholesterol $(<1.03 \mathrm{mM}$ in men, $<1.30 \mathrm{mM}$ in women), hypertension ( $\geq 130 \mathrm{~mm} \mathrm{Hg}$ systolic and/or $\geq 85 \mathrm{~mm} \mathrm{Hg}$ diastolic or antihypertensive drug treatment) and elevated nonfasting plasma glucose $(>7.8 \mathrm{mM}$ or antidiabetic drug treatment). The number of criteria met, the MS score, could take on values from 0 (zero criteria present) to 5 (all criteria present), with values $\geq 3$ defined as MS. Subjects that could not be classified due to missing data in one or more of the five criteria were excluded.

Data were compared across groups using one-way analyses of variance or Fisher's exact test. Associations between exercise volume and intensity and MS risk were investigated by means of logistic regression adjusting for age at baseline and potential confounders. Each of the variables LTPA, jogging and walking speed were adjusted for the two others. All confounders were treated as categorical initially and only as linear variables after testing the assumption of linearity. All initial analyses were sex- 
specific. For the follow-up, data were pooled after initial analyses had shown no gender-related differences in associations with MS. Statistical significance level was set at $\mathrm{p}<0.05$. All analyses were conducted using Stata V.10.1 (Stata Corporation, College Station, Texas, USA).

\section{RESULTS}

\section{Cross-sectional analysis}

The baseline characteristics by level of LTPA are presented in table 1 . Subjects with higher levels of activity were younger and had a more beneficial CVD risk factor profile. Heart rate decreased with increasing level of activity indicating a higher level of cardiorespiratory fitness.

The overall prevalence of MS was 20.7\% in women and $27.3 \%$ in men and closely linked to the LTPA level. In women the prevalence decreased from $31 \%$ in the sedentary to $10.9 \%$ in subjects reporting high level of LTPA. In men, corresponding prevalences were $36.8 \%$ and $13.9 \%$, respectively (table 1 ).

The age adjusted OR for having MS at baseline decreased with increases in LTPA and walking speed and was lower in joggers, whereas association with walking volume was less clear (figure 1). Results were similar in men and women. After multivariable adjustment the associations were only slightly attenuated and remained highly significant with a clear dose-response relationship. Notably, simultaneous adjustment for walking speed and jogging had relatively little effect on risk estimates indicating that each of these three indicators of exercise volume and intensity had independent effect on risk of MS. In the age-adjusted analyses increases in walking volume seemed to lower MS risk in women. However, after multivariable adjustment the association did not remain significant.

\section{Longitudinal analysis}

Only 24 of the 3992 subjects free of MS at baseline who also participated in the fourth survey did not answer the LTPA section of the questionnaires. The baseline characteristics by level of LTPA for the remaining 3968 subjects are outlined in table 2. At the 10-year follow-up, 585 subjects (15.4\%) had developed MS. The MS incidence varied between the different LTPA groups from $19.4 \%$ in the sedentary to $11.8 \%$ in the moderately or highly active. Participants with a high physical activity level were younger and had a more favourable CVD risk factor profile.

Both LTPA, walking speed and jogging conferred a decreased risk of developing MS at follow-up with somewhat weaker associations than found in the crosssectional analyses (figure 2). Multivariable adjustment including mutual adjustment for volume (LTPA) and intensity (walking speed and jogging) attenuated associations slightly (figure 2). Similar to the cross-sectional analyses, increases in walking volume did not convey decreased risk of MS development. Light physical activity less than $2 \mathrm{~h} /$ week was no longer associated with decreased risk of MS (OR 0.99 (0.71 to 1.38)).

\section{DISCUSSION}

We found a decreased risk of developing MS with higher walking speed, jogging and high levels of LTPA in both men and women whereas walking volume and light physical activity were not associated with MS development. Our data indicated that the risk of developing MS may be reduced as much as $35-50 \%$ in subjects who are more physically active.

MS is highly prevalent worldwide ${ }^{25} 26$ and MS incidence is considered epidemic in nature. ${ }^{13}$ In the present study population of 21-98-year-old Danish men and women living in Copenhagen in 1994, the average MS prevalence was $21 \%$ in women and $27 \%$ in men. In a similar Swedish study MS prevalence was $19 \%$ in women and $24 \%$ in men. ${ }^{6}$ Even higher prevalences have been reported, ${ }^{7}{ }^{13}$ but direct comparisons between studies are obviously difficult due to differences in study population characteristics and choice of MS definitions and diagnostic criteria. Cumulated MS incidence during the 10-year follow-up was $15.4 \%$, ranging from $19.4 \%$ in the sedentary group to $11.8 \%$ in the moderately to highly active group. In a meta-analysis by Ford and $\mathrm{Li}^{13}$ incidences of $10.4-17.5 \%$ were reported with follow-up time ranging from 4 to 23 years. Thus MS constitutes an increasing problem with important economic and public health implications.

Our results confirm the association between physical activity and MS risk found in earlier cross-sectional studies. ${ }^{3} 6{ }^{6} 12 \quad 13$ However, several longitudinal studies have failed to demonstrate an association between physical activity and MS incidence, suggesting that a low LTPA level could be the result of MS, rather than its cause. ${ }^{9}{ }^{17}$ In a population of 862 , 50-year-old men free of diabetes and MS it was found that BMI and selfreported LTPA were independent predictors of insulin resistance at 20-year follow-up, indicating that obesity and physical inactivity could increase insulin resistance by independent pathways. ${ }^{9}$ However, only BMI-and not LTPA—was a significant predictor of MS at follow-up. Correspondingly, a study of 714 White, Black and Hispanic men and women without MS at baseline followed for an average of 5.2 years found that self-reported physical activity at baseline did not predict MS status at follow-up. ${ }^{17}$ In the CCHS, Berentzen et $a l^{27}$ demonstrated that in 4808 men and women aged 21-81 years, LTPA did not have a significant effect on changes in waist circumference-a key component in the MSduring a 10-year follow-up period. In contrast to this, a 15-year follow-up study of 4192 young (18-30 years of age) adult men (45\%) and women $(55 \%)$ showed that physical activity was inversely associated with MS risk. ${ }^{28}$ However, after multivariable adjustment the association was only significant when comparing the highest and lowest physical activity groups (OR 0.65). This finding 
Table 1 Anthropometric, metabolic and lifestyle characteristics of the participants in the third examination of the CCHS, 1991-1994, by LTPA

\section{LTPA women $(\mathrm{n}=5608)$}

\section{Sedentary}

Light

( $\mathrm{n}=754)$

\section{Age (years)}

BMI $\left(\mathrm{kg} / \mathrm{m}^{2}\right)$

Waist $(\mathrm{cm})$

$64.5( \pm 15.0)$

( $n=3277)$

SBP $(\mathrm{mm} \mathrm{Hg})$ $26.7( \pm 5.6)$

$60.0( \pm 14.8)$

Moderate

$60.0( \pm 14.8) \quad 55.0( \pm 15.5)$

$55.0( \pm 15.5)$
$24.4( \pm 4.0)$

$82.9( \pm 12.1) \quad 79.8( \pm 11.1)$

$87.3( \pm 13.7)$

$\mathrm{DBP}(\mathrm{mm} \mathrm{Hg})$

$141.9( \pm 23.5)$

$139.4( \pm 24.4) \quad 133.8( \pm 22.8)$

$\mathrm{HDL}(\mathrm{mM})$

Glu (mM)

TG (mM)

Current

$82.7( \pm 12.8)$

$1.64( \pm 0.52)$

$5.99( \pm 1.98)$

$83.1( \pm 12.1) \quad 81.6( \pm 11.8)$

$1.71( \pm 0.49) \quad 1.79( \pm 0.49)$

$5.66( \pm 1.67) \quad 5.46( \pm 1.29)$

$1.71( \pm 0.95) \quad 1.55( \pm 0.9)$

$384(50.9)$

1495 (45.6)

630 (42.9)

smoking (n (\%))

Alcohol (units/

week)

Heart rate (bpm)

Jogging (n (\%))

High walking

speed (n (\%))

Walking vol. $>1 \mathrm{~h} /$

day $(\mathrm{n}(\%))$

MS (n (\%))

$5.1( \pm 9.0)$

$5.4( \pm 7.1)$

$6.5( \pm 7.6)$

$74.9( \pm 12.3)$

$2(0.3)$

$73.3( \pm 12.4) \quad 71.6( \pm 11.8)$

$50(1.5)$

$128(8.7)$

68 (9.6)

$602(18.5)$

519 (35.6)

200 (26.67)

$1715(52.48) \quad 918(62.58)$

$214(31.0)$

$690(21.8)$

$203(14.1)$

High

( $n=108)$

LTPA men $(n=4382)$

Values are mean ( $(\mathrm{SD})$

BMI, body mass index; DBP, diastolic blood pressure; Glu, glucose; HDL, high-density lipoprotein; LTPA, leisure time physical activity; MS, metabolic syndrome; SBP, systolic blood pressure; TG, triglyceride; Walking vol., walking volume. 


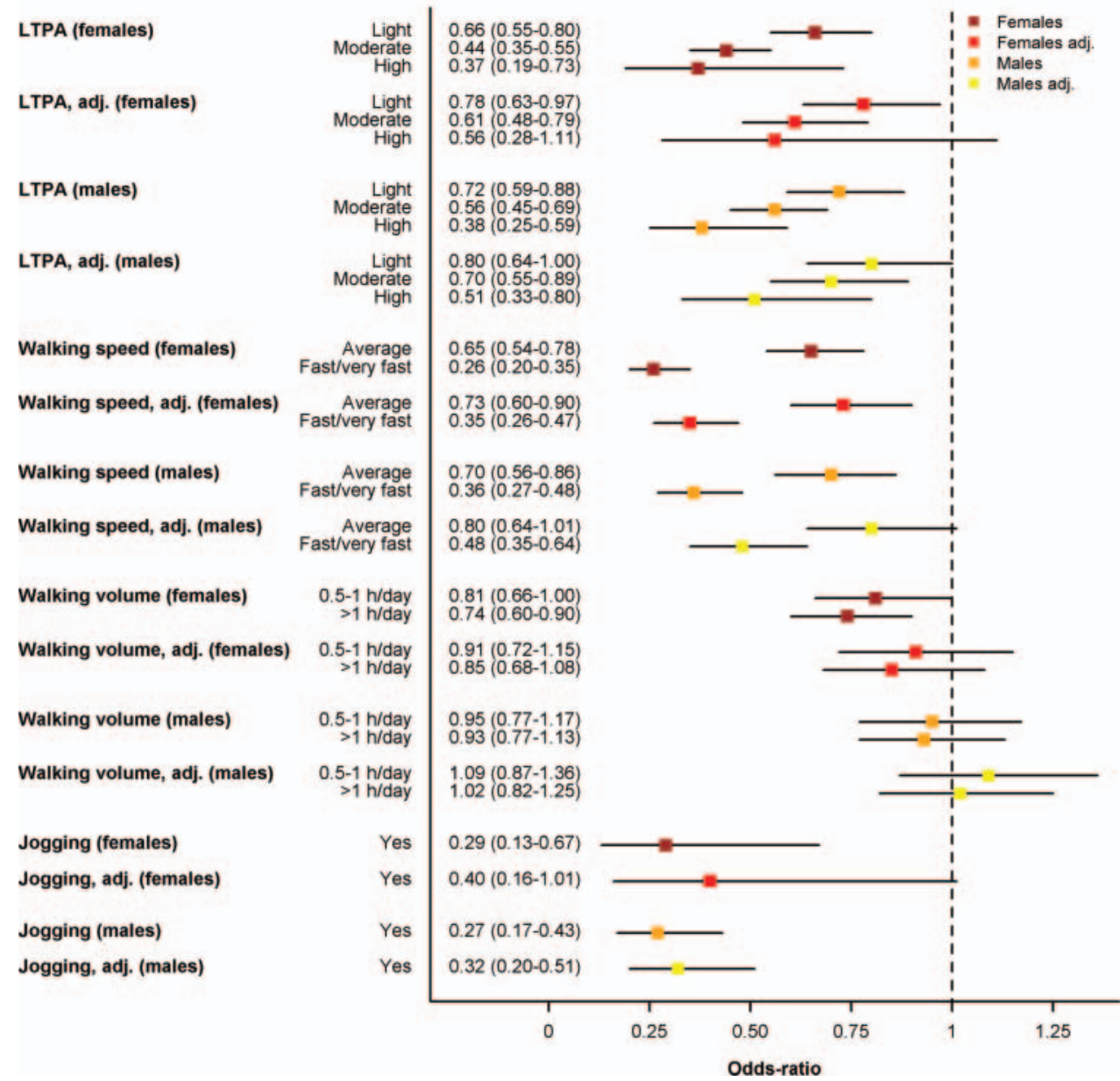

Figure 1 Cross-sectional OR for MS. Age-adjusted and multivariable-adjusted ORs for MS risk by level of LTPA level, jogging and walking speed and volume at baseline 1991-1994. Reference groups are sedentary, slow walking speed, walking less than $0.5 \mathrm{~h} /$ week and no jogging, respectively. Multivariable adjusted models included LTPA, jogging, walking speed and volume, alcohol, smoking, income, duration of schooling, education, cohabitation and age. adj., adjusted; LTPA, leisure time physical activity; MS, metabolic syndrome.

suggests that there is a threshold below which physical activity does not confer protection against MS. In concordance with this, our longitudinal analyses showed that subjects who were physically active $2-4 \mathrm{~h}$ /week but who did not engage in physical activity with more intensity (fast walking, jogging) were not protected against the development of the MS. Interestingly, this finding also seems to apply to high-risk populations. A recent study including 486 middle-aged men and women at high risk of developing type 2 diabetes found that increases in moderate-to-vigorous LTPA reduced MS risk and could even lead to its resolution in individuals already diagnosed with the MS. ${ }^{18}$ Changes in lowintensity LTPA did not affect MS development or resolution.

Our analyses did not indicate any evidence of a beneficial effect of increasing walking volume whereas walking speed and jogging both reduced MS risk after adjusting for volume of physical activity (LTPA), thus supporting the perception that intensity plays a key role in MS prevention. In a cohort of 612 middle-aged men with assessment of both LTPA and $\mathrm{VO}_{2 \max }$, MS risk was lower at 4-year follow-up in men engaging in $>3 \mathrm{~h}$ /week of moderate or vigorous LTPA and in men in the upper $\mathrm{VO}_{2 \max }$ tertile, ${ }^{15}$ again pointing to an effect of the intensity of physical activity. In a cross-sectional study of 1069 middle-aged men, cardiorespiratory fitness was inversely associated with MS whereas there was no effect of lowintensity activity. ${ }^{29}$ Other studies have confirmed cardiorespiratory fitness as a predictor of less progression towards the $\mathrm{MS}^{7}{ }^{10}$ even in high-risk individuals. ${ }^{10}$ Similarly, Hassinen $e t a l^{19}$ found that high levels of cardiorespiratory fitness decreased the risk of developing MS and could even increase the probability of MS resolution in a population of 1226 men and women aged $57-78$ years. In the CCHS, Schnohr et $a l^{20}{ }^{30}$ have previously shown that both jogging and walking speed rather than duration were protective of all-cause and CVD mortality. A recent meta-analysis of walking volume and pace on risk of coronary heart disease suggested a more 
Table 2 Anthropometric, metabolic and lifestyle characteristics of the subjects without MS at baseline in 1991-1994 who participated at follow-up in 2001-2004, by LTPA

\begin{tabular}{|c|c|c|c|c|}
\hline LTPA $(n=3968)$ & Sedentary $(n=296)$ & Light $(n=2028)$ & Moderate+high $(n=1644)$ & p Value \\
\hline Women (\%) & 61.1 & 65.9 & 53.0 & \\
\hline Age (years) & $51.8( \pm 13.7)$ & $53.2( \pm 13.3)$ & $50.2( \pm 14.7)$ & $<0.0001$ \\
\hline Height (cm) & $168.5( \pm 10.1)$ & $168.4( \pm 9.1)$ & $170.8( \pm 9.5)$ & $<0.0001$ \\
\hline Weight (kg) & $70.9( \pm 13.5)$ & $69.4( \pm 12.1)$ & $70.4( \pm 11.9)$ & 0.018 \\
\hline BMI $\left(\mathrm{kg} / \mathrm{m}^{2}\right)$ & $24.9( \pm 4.0)$ & $24.5( \pm 3.5)$ & $24.1( \pm 3.1)$ & $<0.0001$ \\
\hline Waist (cm) & $85.6( \pm 11.7)$ & $83.0( \pm 11.0)$ & $82.5( \pm 10.7)$ & $<0.0001$ \\
\hline $\mathrm{SBP}(\mathrm{mm} \mathrm{Hg})$ & $130.5( \pm 18.5)$ & $132.1( \pm 20.6)$ & $130.7( \pm 20.0)$ & 0.090 \\
\hline $\mathrm{DBP}(\mathrm{mm} \mathrm{Hg})$ & $81.8( \pm 11.6)$ & $82.4( \pm 11.9)$ & $81.9( \pm 11.8)$ & 0.35 \\
\hline HDL (mM) & $1.61( \pm 0.46)$ & $1.69( \pm 0.46)$ & $1.68( \pm 0.46)$ & 0.022 \\
\hline Glu (mM) & $5.42( \pm 1.16)$ & $5.38( \pm 0.92)$ & $5.32( \pm 0.88)$ & 0.073 \\
\hline TG (mM) & $1.56( \pm 0.84)$ & $1.50( \pm 0.79)$ & $1.43( \pm 0.76)$ & 0.0065 \\
\hline Current smoking (n (\%)) & $166(56.1)$ & $916(45.2)$ & $658(40.1)$ & $<0.0001$ \\
\hline Alcohol (units/week) & $10.9( \pm 14.1)$ & $8.7( \pm 10.1)$ & $9.7( \pm 10.2)$ & 0.0004 \\
\hline Heart rate (bpm) & $71.6( \pm 11.8)$ & $71.2( \pm 11.5)$ & $68.5( \pm 11.7)$ & $<0.0001$ \\
\hline Jogging (n (\%)) & $7(2.4)$ & $71(3.5)$ & $274(16.7)$ & $<0.0001$ \\
\hline High walking speed (n (\%)) & 69 (23.5) & $545(27.0)$ & $651(39.8)$ & $<0.0001$ \\
\hline Walking volume >1 h/day (n (\%)) & $92(31.08)$ & $999(49.38)$ & $952(57.94)$ & $<0.0001$ \\
\hline MS at CCHS 4 (n (\%)) & $53(19.4)$ & $337(17.6)$ & $187(11.8)$ & $<0.0001$ \\
\hline
\end{tabular}

Values are mean ( \pm SD)

BMI, body mass index; CCHS, Copenhagen City Heart Study; DBP, diastolic blood pressure; Glu, glucose; HDL, high-density lipoprotein; LTPA, leisure time physical activity; MS, metabolic syndrome; SBP, systolic blood pressure; TG, triglyceride; walking vol., walking volume.

pronounced dose-response protection effect for walking pace than walking volume. ${ }^{21} \mathrm{~A}$ similar meta-analysis by Hamer and Chida ${ }^{22}$ regarding the effect of walking on cardiovascular disease and all-cause mortality found that walking pace was a stronger predictor of overall risk than walking volume. Although the present study did not point towards any association between duration of walking and MS risk, potential health benefits could still ensue from increasing walking volume. Regardless, it seems that increasing walking intensity rather than

OR for MS risk, longitudinal

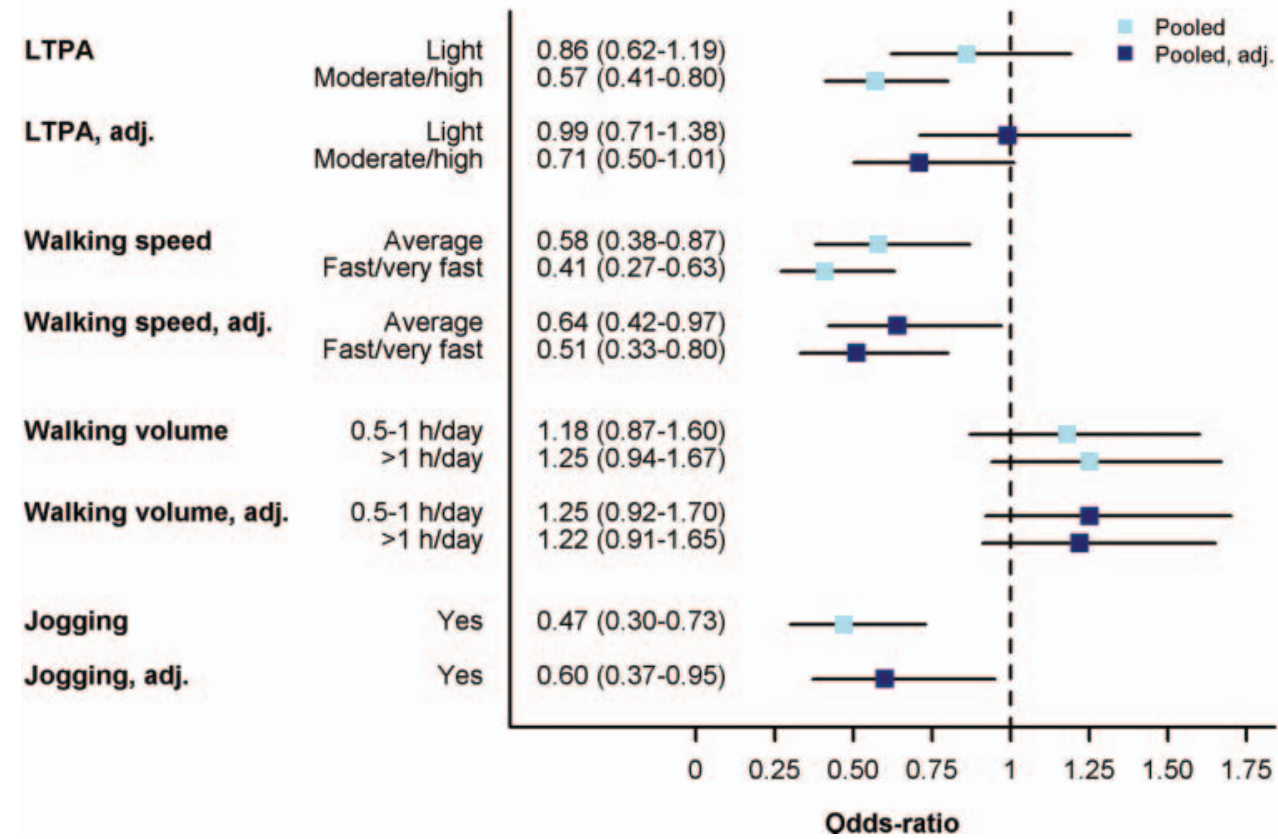

Figure 2 Longitudinal analysis of risk of MS. Age-adjusted and gender-adjusted and multivariate-adjusted ORs for MS risk based on differences in LTPA level, jogging and walking speed and volume. Reference groups are sedentary, slow walking speed, walking less than $0.5 \mathrm{~h} /$ week and no jogging, respectively. Multivariable-adjusted models included LTPA, jogging, walking speed and volume, alcohol, smoking, income, duration of schooling, education, cohabitation and age. adj., adjusted; LTPA, leisure time physical activity; MS, metabolic syndrome. 
walking volume would be the sensible strategy in attempt to reduce MS risk.

The primary strength of the present study is the considerable study population size that represents a random sample of the Copenhagen population making our results applicable to the general population. The longitudinal study design enables us to investigate the causal inference between physical activity and the MS. Combining a longitudinal study design with a large study population provides useful information about the relationship between physical activity and MS risk. Weaknesses of our study include the use of selfadministered questionnaires to assess LTPA level, which may result in poorer precision compared to more objective measures of physical activity. However, earlier studies have shown that self-administered questionnaires can produce reliable data when estimating habitual physical activity. ${ }^{31} 32$ Our data do not include direct fitness level measurements or exact information regarding volume and intensity of physical activity, but it seems plausible that the combined information on jogging, walking speed and physical activity habits yield a fair estimate of volume as well as intensity. The study comprised relatively few joggers and the questionnaires used did not take jogging intensity into consideration. As a result of this we could not draw any conclusions regarding the effect of jogging intensity versus jogging volume on MS risk.

Furthermore, the questionnaires did not provide any information regarding diet or nutrition. Obviously, potential differences in dietary habits between the LTPA groups could have significant impact on the results of the present study. The use of a modified cut-off level for plasma-glucose in consequence of the lack of fastingplasma glucose measurements is unlikely to have introduced a significant bias towards an overestimation of patients with MS as the cut-off level deliberately was above levels normally seen in individuals with normal glucose metabolism.

\section{PERSPECTIVE}

Significantly lower risk of MS was found in the moderately and highly physically active groups compared to their sedentary counterparts whereas light physical activity and even more than $1 \mathrm{~h}$ of walking daily did not confer protection against MS. Participation in regular moderate or vigorous activity such as brisk walking could improve cardiorespiratory fitness and decrease MS risk. If results can be corroborated by intervention studies showing beneficial cardiovascular effects of increased walking speed, this may represent a low-cost intervention with minimal side effects and numerous significant health benefits.

Contributors EP, OPK and PS all took part in data research and furthermore contributed to the discussion and reviewed/edited the manuscript. JLM researched data and reviewed/edited the manuscript. AHL researched data and wrote the manuscript.
Competing interests None.

Ethics approval National Board of Ethics.

Provenance and peer review Not commissioned; externally peer reviewed.

Data sharing statement No additional data are available.

\section{REFERENCES}

1. Grundy SM, Cleeman JI, Daniels SR, et al. Diagnosis and management of the metabolic syndrome. Circulation 2005;112: e285-90.

2. Alberti KGMM, Eckel RH, Grundy SM, et al. Harmonizing the metabolic syndrome. Circulation 2009;120:1640-5.

3. Rennie KL, McCarthy N, Yazdgerdi S, et al. Association of the metabolic syndrome with both vigorous and moderate physical activity. Int J Epidemiol 2003;32:600-6.

4. Lakka TA, Laaksonen DE. Physical activity in prevention and treatment of the metabolic syndrome. Appl Physiol Nutr Metab 2007;32:76-88.

5. Sundström J, Risérus U, Byberg L, et al. Clinical value of the metabolic syndrome for long term prediction of total and cardiovascular mortality: prospective, population based cohort study. Br Med J 2006;332:878-82.

6. Halldin M, Rosell M, de Faire U, et al. The metabolic syndrome: prevalence and association to leisure-time and work-related physical activity in 60-year-old men and women. Nutr Metab Cardiovasc Dis 2007; 17:349-57.

7. Carrol S, Dudfield M. What is the relationship between exercise and metabolic abnormalities - a review of the metabolic syndrome. Sports Med 2004;34:371-418.

8. Sundström J, Vallhagen E, Risérus $U$, et al. Risk associated with the metabolic syndrome versus the sum of its individual components. Diabetes Care 2006;29:1673-4.

9. Ingelsson E, Ärnlöv J, Sundström J, et al. Relative importance and conjoint effects of obesity and physical inactivity for the development of insulin resistance. Eur J Card Prev Rehabil 2009;16:28-33.

10. LaMonte MJ, Barlow CE, Jurca R, et al. Cardiorespiratory fitness is inversely associated with the incidence of metabolic syndrome. Circulation 2005;112:505-12.

11. Gaesser GA. Exercise for prevention and treatment of cardiovascular disease, type 2 diabetes and metabolic syndrome. Curr Diab Rep 2007;7:14-19.

12. Duncan GE. Exercise, fitness and cardiovascular disease risk in type 2 diabetes and the metabolic syndrome. Curr Diab Rep 2006;6:29-35.

13. Ford ES, Li C. Physical activity or fitness and the metabolic syndrome. Expert Rev Cardiovasc Ther 2006;4:897-915.

14. Petersen L, Schnohr P, Sørensen TIA. Longitudinal study of the long-term relation between physical activity and obesity in adult. Int $\mathrm{J}$ Obes 2004;28:105-12.

15. Laaksonen DE, Lakka HM, Salonen JT, et al. Low levels of leisure-time physical activity and cardiorespiratory fitness predict development of the metabolic syndrome. Diabetes Care 2002;25:1612-18.

16. Ekelund U, Brage S, Franks PW, et al. Physical activity energy expenditure predicts progression toward the metabolic syndrome independently of aerobic fitness in middle-aged healthy caucasians -The Medical Research Council Ely Study. Diabetes Care 2005;28:1195-200.

17. Palaniappan L, Carnethon MR, Wang Y, et al. Predictors of the incident metabolic syndrome in adults-The Insulin Resistance Atherosclerosis Study. Diabetes Care 2004;27:788-93.

18. Ilanne-Parikka P, Laaksonen DE, Eriksson JG, et al. Leisure-time physical activity and the metabolic syndrome in the Finnish diabetes prevention study. Diabetes Care 2010;33:1610-17.

19. Hassinen M, Lakka TA, Hakola L, et al. Cardiorespiratory fitness and metabolic syndrome in older men and women: the Dose Responses to Exercise Training (DR's EXTRA) study. Diabetes Care 2010;33:1610-17.

20. Schnohr $P$, Scharling $H$, Jensen JS. Intensity versus duration of walking, impact on mortality: the Copenhagen City Heart Study. Eur $J$ Card Prev Rehabil 2007;14:72-8.

21. Zheng H, Orsini N, Amin J, et al. Quantifying the dose-response of walking in reducing coronary heart disease risk: meta-analysis. Eur $J$ Epidemiol 2009;24:81-192.

22. Hamer M, Chida Y. Walking and primary prevention: a meta-analysis of prospective cohort studies. Br J Sports Med 2008;42:238-43. 
23. Saltin B, Grimby G. Physiological analysis of middle-aged and old former athletes: comparison with still active athletes of the same ages. Circulation 1968;38:1104-15.

24. Nordestgaard BG, Hilsted L, Stender S. Plasmalipider hos ikke fastende patienter og signalværdier på laboratoriesvar. Ugeskr Læger 2009;171:1093.

25. Ford ES, Giles WH, Dietz WH. Prevalence of the metabolic syndrome among us adults. JAMA 2002;287:356-9.

26. Cameron AJ, Shaw JE, Zimmet PZ. The metabolic syndrome: prevalence in worldwide populations. Endocrinol Metab Clin North Am 2004;33:351-75.

27. Berentzen $\mathrm{T}$, Petersen L, Schnohr $\mathrm{P}$, et al. Physical activity in leisure-time is not associated with 10-year changes in waist circumference. Scand J Med Sci Sports 2008;18:719-27.
28. Carnethon MR, Loria CM, Hill JO, et al. Risk factors for the metabolic syndrome - the coronary artery risk development in young adults (Cardia) study, 1985-2001. Diabetes Care 2004;27:2707-15.

29. Lakka TA, Laaksonen DE, Lakka H-M, et al. Sedentary lifestyle, poor cardiorespiratory fitness, and the metabolic syndrome. Med Sci Sports Exerc 2003;35:1279-86.

30. Schnohr P, Parner J, Lange P. Mortality in joggers: population based study of 4658 men. Br Med J 2000;321 602-3.

31. Lichtman SW, Pisarska K, Berman ER, et al. Discrepancy between self-reported and actual caloric intake and exercise in obese subjects. N Engl J Med 1992;327:1893-8.

32. Wolf AM, Hunter DJ, Colditz GA, et al. Reproducibility and validity of a self-administered physical activity questionnaire. Int $J$ Epidemiol 1994;23:991-9. 